\title{
Microfabrication of Linear Translator Tuning Elements in Submillimeter-Wave Integrated Circuits
}

\author{
Victor M. Lubecke, Senior Member, IEEE, William R. McGrath, Member, IEEE, \\ Yu-Chong Tai, and David B. Rutledge, Fellow, IEEE
}

\begin{abstract}
A micromechanical planar tuning element has been developed and demonstrated in a fully monolithic $620-\mathrm{GHz}$ integrated circuit. It allows for the mechanical variation of the electrical length of a coplanar transmission line tuner and is called a sliding planar backshort (SPB). It consists of a movable patterned rectangular metal plate confined by polyimide flanges along two of its edges to allow guided linear translation along the length of a dielectric-coated coplanar transmission line. Its fabrication involves an application of sacrificial-layer and molding techniques to materials and processes which are compatible with the fabrication of a wide range of submillimeter-wave integrated circuits. This is the first reported micromechanically adjustable tuning element demonstrated at submillimeter wavelengths. [319]
\end{abstract}

Index Terms - Backshort, coplanar, MEMS, micromachining, millimeter wave, monolithic integrated circuit, planar tuning element, submillimeter wave.

\section{INTRODUCTION}

$\mathbf{M}$ ICROMACHINING techniques offer great potential to millimeter- and submillimeter-wave circuit technology. Critical dimensions in these circuits decrease with increasing operating frequency, creating fabrication difficulties which can be addressed through micromachining. Additionally, micromachining techniques and micromechanical components can allow for the development of new unconventional highfrequency circuitry which can offer superior performance.

Silicon bulk micromachining techniques have already been demonstrated in submillimeter- and near-submillimeter-wave circuits. Reflecting cavities for membrane-supported planar submillimeter-wave antennas have been fabricated by stacking anisotropically etched silicon wafers [1]. A similar selective etching technique has also been employed to create waveguide sections in silicon, incorporating internally suspended membranes which can allow for the integration of planar high-frequency devices [2]. Bulk techniques have also been

Manuscript received December 29, 1997; revised August 3, 1998. This work was supported in part by the Innovative Science and Technology Office of the Ballistic Missile Defense Organization, Office of Space Science and Technology, National Aeronautics and Space Administration (NASA), and a NASA GSRP Fellowship. Subject Editor, N. de Rooij.

V. M. Lubecke was with the Division of Engineering and Applied Science, California Institute of Technology, Pasadena, CA 91125 USA. He is now with the Photodynamics Research Center, Institute of Physical and Chemical Research (RIKEN), Sendai 980, Japan (e-mail: victor@postman.riken.co.jp).

Y.-C. Tai and D. B. Rutledge are with the Division of Engineering and Applied Science, California Institute of Technology, Pasadena, CA 91125 USA.

W. R. McGrath is with the Center for Space Microelectronics Technology, Jet Propulsion Laboratory, California Institute of Technology, Pasadena, CA 91109 USA.

Publisher Item Identifier S 1057-7157(98)08920-3. used to selectively remove substrate material from critical regions in planar high-frequency transmission line components in order to reduce losses and enhance isolation [3]. Silicon surface micromachining techniques offer potential in this field as well, as they have been used to create various submillimeter-scale rotating and translating structures [4] on which mechanically adjustable submillimeter-wave components can be based. Techniques similar to those demonstrated in simplified LIGA-like processes [5] can potentially make surface micromachining techniques practical in a variety of conventional high-frequency circuits.

At millimeter and submillimeter wavelengths, the performance of common semiconducting and superconducting devices is severely degraded by the effects of parasitic reactances inherent in their geometries [6], [7]. These effects are not easily characterized, and adjustable impedance matching circuits are typically needed to make practical use of such devices. A common approach is to embed the device in a waveguide circuit and employ mechanically adjustable waveguide backshorts as tuning elements which serve to optimize the device performance by compensating for the parasitic reactance [8]. The critical dimensions for these circuits are very small, decreasing in size as the frequency of interest increases. This makes fabrication of such waveguide circuits exceedingly costly and difficult and has motivated interest in alternative planar approaches.

Monolithic integrated-circuit technology promises a practical means for realizing reliable and reproducible planar millimeter- and submillimeter-wave circuits. Planar circuits are fabricated through photolithographic techniques, which allow for the cost-effective production of intricate designs not possible with waveguide technology. These circuits, however, must also provide compensation for parasitic device reactances. Conventional planar technology allows for only fixed tuning elements, providing no means for postfabrication optimization of performance [9]. This makes characterization of the component elements critical, and it is not usually possible to achieve satisfactory results without multiple design and fabrication iterations. It would be desirable to incorporate in these planar circuits the same kind of mechanically adjustable tuning available in waveguide circuits.

An adjustable planar tuning element, which functions in a planar circuit analogously to a backshort in a waveguide circuit, has been developed along with a process for its fabrication as an integral part of a millimeter- or submillimeter-wave monolithic circuit. This is the first reported demonstration of an integrated micromechanically adjustable tuning element 


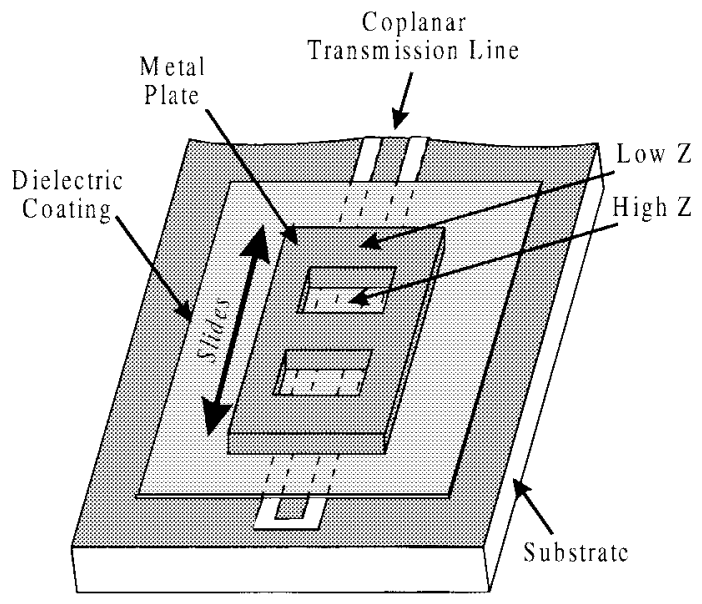

Fig. 1. Conceptual illustration of an SPB tuning element. A patterned metal plate slides over a dielectric-coated planar transmission line to vary the electrical length.

at submillimeter wavelengths. The tuning element, called a sliding planar backshort (SPB), is formed as an integral part of a coplanar transmission line tuning stub, using only conventional micron-scale fabrication techniques commonly employed for submillimeter-wave circuits, which include UV lithography, evaporated and electroplated metals, and sputtered and spun-on dielectrics. The SPB can be used in developmental integrated circuits as an aid for device characterization or as a means to optimize in-use performance for a variety of submillimeter-wave integrated circuits.

\section{INTEGRATED SPB's}

An SPB consists of a rectangular metal plate, with appropriately sized and spaced holes, which rests on top of a dielectric-coated planar transmission line, as shown in Fig. 1. The impedance of the sections of line covered by metal is greatly reduced, while the uncovered sections retain their higher impedance. Each of these sections is approximately one quarter of a wavelength long, and the cascade of alternating low- and high-impedance sections results in an extremely lowimpedance termination. This termination can be moved to vary the electrical length of a planar transmission line tuning stub by simply sliding the metal plate along the length of the line. Such adjustable tuning stubs can be used in a variety of ways to adjust the impedance match between the various elements of a circuit [10]. The semiempirical design of the SPB was originally carried out with a 2-GHz-scale model [11], and SPB tuners have since been successfully demonstrated as discrete components at frequencies up to $100 \mathrm{GHz}$ [12].

The wavelength for a signal guided on a planar transmission line is determined by the frequency of the signal and the dielectric properties of the substrate, decreasing in size with increasing frequency and dielectric constant [13]. Since an SPB tuner works as a distributed transmission line component, its dimensions vary accordingly. At low frequencies, these dimensions are large by micromachining standards, even for substrate materials with relatively high-dielectric constants like silicon. At frequencies above $100 \mathrm{GHz}$, however, the dimensions can be on the order of hundreds or tens of microns.
At $620 \mathrm{GHz}$, an SPB on a silicon dioxide substrate can be about $200 \mu \mathrm{m}$ wide, comparable in size to linear translating structures fabricated through the surface micromachining of silicon [4]. Unfortunately, the processes and materials typically used for such structures can be inappropriate for, or incompatible with, those often needed in submillimeter-wave circuit fabrication.

Micromachining with polysilicon components and silicon dioxide sacrificial layers typically requires high-temperature chemical vapor deposition (CVD) or furnace growth and aggressive and hazardous chemical etchants. Many conventional submillimeter-wave circuits, containing delicate thinfilm structures, diodes, and other devices, cannot easily accommodate such processes. It is desirable that the sliding element of an SPB tuner be made from a good conductor through a process which involves temperatures and reactions which can be safely used on a wide range of insulating substrates which already contain thin-film circuitry. Fortunately, key features from the silicon-based technique can be suitably combined with an LIGA-like UV process to allow for the fabrication of SPB tuners in a variety of submillimeter-wave circuit applications, using only rudimentary fabrication facilities and very low-hazard materials and processes [14].

An all-monolithic quasi-optical $620-\mathrm{GHz}$ direct-detection circuit was developed to demonstrate the operation of integrated submillimeter-wave SPB tuners. This circuit uses a dielectric-filled parabola [15] substrate lens to focus radiation onto a slot antenna and couples this radiation to a detector by means of two coplanar waveguide (CPW) transmission lines, each with integrated SPB tuners. One SPB tuner creates a variable reactance in series between the antenna and the detector, potentially serving to compensate for any unwanted reactance when the slot is not resonant. The other SPB tuner creates a variable susceptance in parallel with the detector and could be used to compensate for the parasitic capacitance found in some otherwise desirable submillimeter-wave devices. The entire circuit can be fabricated through simple processes commonly employed in the making of millimeterand submillimeter-wave integrated circuits.

\section{Submillimeter-WAVE Circuit FABrication}

Submillimeter-wave circuits are made with a variety of lowloss dielectric substrates, high-speed semiconductor devices, and even superconducting thin films. The choices depend on the applications, and components which are not restricted to one medium or process are desirable.

Except for the SPB tuners, the 620-GHz detector circuit used here is conventional; it incorporates materials, components, and fabrication processes common to many fixedtuned submillimeter-wave circuits. Fused quartz was used for the substrate lens, which provides low-loss transmission line properties and a reasonable match to free space. The transmission line and antenna circuitry were straightforward thin-film patterns, and a patterned thin film of bismuth was used as a thermoelectric detector [16]. While this type of detector does not provide the sensitivity of semiconducting and superconducting mixing elements, it offers the advan- 


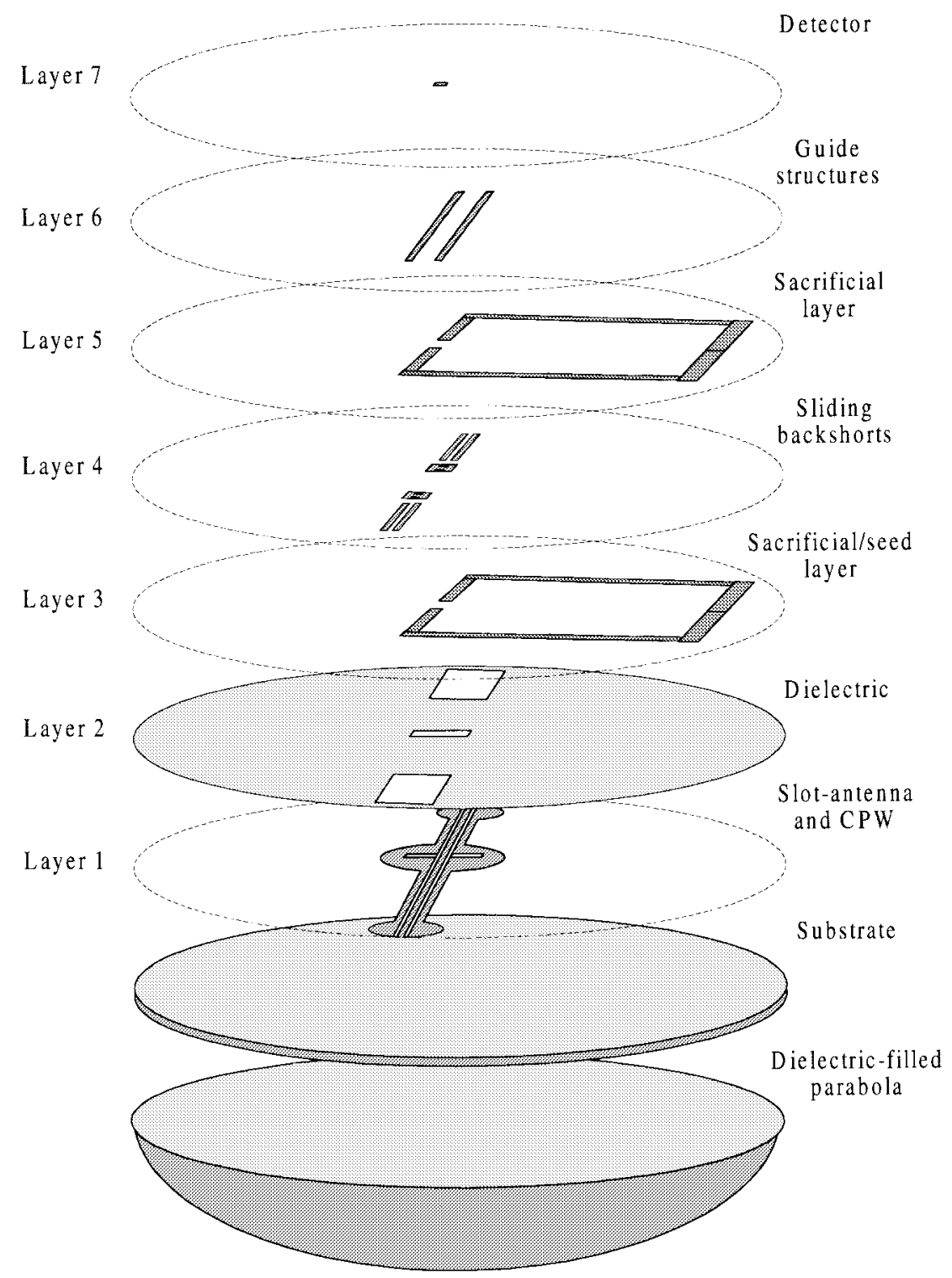

Fig. 2. Exploded illustration of the seven-layer circuit. Two SPB tuners were included using conventional submillimeter-wave circuit fabrication techniques.

tage of working at room temperature, with only a single layer of processing. Techniques are available, however, for integrating gallium arsenide Schottky diodes, superconductor-insulator-superconductor (SIS) junctions, and similar devices in this circuit [17], [18]. The completed circuit required seven masks and seven processing layers, and the process is illustrated in Fig. 2.

The entire circuit was fabricated at the center of a round $254-\mu$ m-thick 19-mm-diameter fused-quartz wafer which could be seamlessly installed on a quartz lens. The first process layer consisted of $1000 \AA$ of gold, with $70-\mu \mathrm{m}$ chrome adhesion layers above and below, deposited by electron-beam evaporation and etched to form the slots for the antenna and CPW transmission lines. Since the $620-\mathrm{GHz}$ signal must pass through the circuit in order to utilize the substrate lens, excess metal was also etched, leaving only enough in the vicinity of the transmission lines and antenna to serve as a proper ground plane. A $1000-\AA ̊$ layer of silicon dioxide was then applied to provide mechanical and dc isolation between the CPW lines and subsequent layers. The silicon dioxide was deposited on the circuit by low-temperature radio frequency $(\mathrm{RF})$ sputtering using a photoresist lift-off stencil to define small openings which would allow the thin-film detector and bias wire bonds to make ohmic contact to the CPW lines beneath it. Two SPB tuning elements were then added by the process described in the following section. The final processing layer consisted of a thermally evaporated 6000 - $\AA$-thick bismuth film patterned with a photoresist lift-off stencil.

\section{SPB TUNER FABRICATION}

The procedure used for fabricating the micromechanical SPB tuners on the dielectric-coated CPW lines is illustrated in Fig. 3. The processing steps are not so different from those for capacitors and air bridges in fixed tuned circuits, but produce a more versatile tuning element. Thus, it was possible for this entire circuit to be fabricated with low-hazard processes common to high-frequency circuit fabrication without the use of an environmentally controlled clean room [19], [20]. 

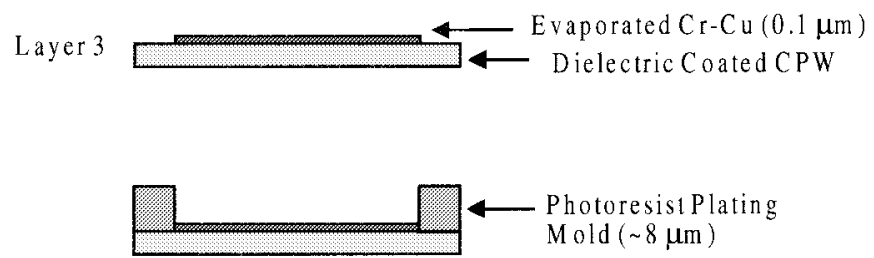

Layer 4 Plated $\mathrm{Au}(\sim 5 \mu \mathrm{m})$

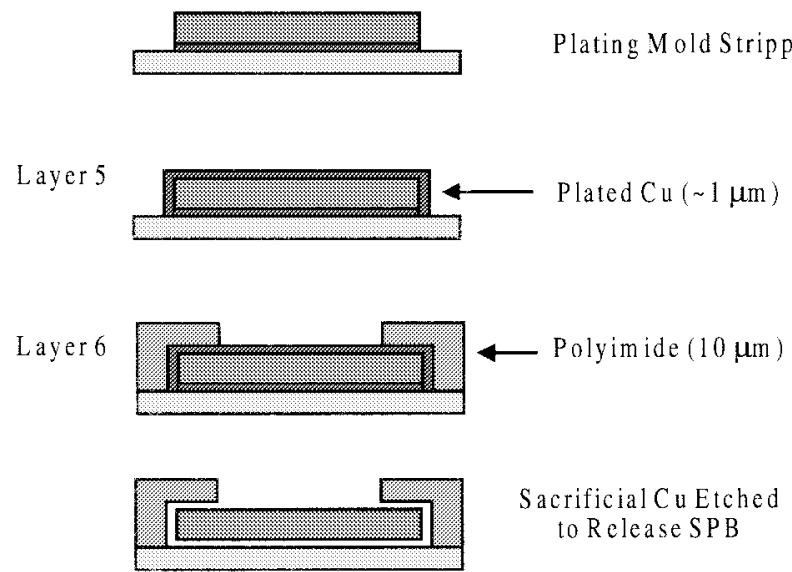

Fig. 3. Simplified illustration of the SPB fabrication process. Sacrificial layers are used to form an SPB which is constrained by polyimide guide structures.

A photoresist lift-off stencil was applied and patterned to define a sacrificial-seed layer. This pattern consisted of rectangular strips over each CPW line, each as wide as the SPB $(200 \mu \mathrm{m})$ and extending to the edge of the substrate to allow for electrode connection. This third layer was formed by depositing a $1700-\AA ̊$ layer of copper over a $70-\AA$ chrome adhesion layer through electron-beam evaporation and then lifting the stencil and unwanted film in acetone.

Next, an 8- $\mu$ m layer of photoresist was applied to the circuit, patterned to form a mold layer, and hard baked. This layer defined the shape of each SPB, several sacrificial pieces used to define the region into which each SPB would slide, and two one-square-mm patches. These patterns were all formed over the sacrificial-seed layer to allow for electroplating, with the large square patches serving to increase the plating area and allow for the use of an easily maintained dc plating current. The wafer was then dipped in gold electroplating solution where $5-\mu \mathrm{m}$-thick patterned gold structures were plated within the mold, forming the fourth circuit layer.

The mold layer was then striped in N-methyl 2-pyrrolidone, and a $1-\mu \mathrm{m}$ sacrificial copper coating was applied to the exposed SPB and sacrificial structures by connecting the circuit to an electrode and immersing it in copper electroplating solution. This formed the fifth circuit layer. A $13-\mu \mathrm{m}$-thick layer of photosensitive polyimide precursor was then spun onto the circuit and UV patterned to form two digitated strips, each overlapping a side of the copper-coated gold structures. The

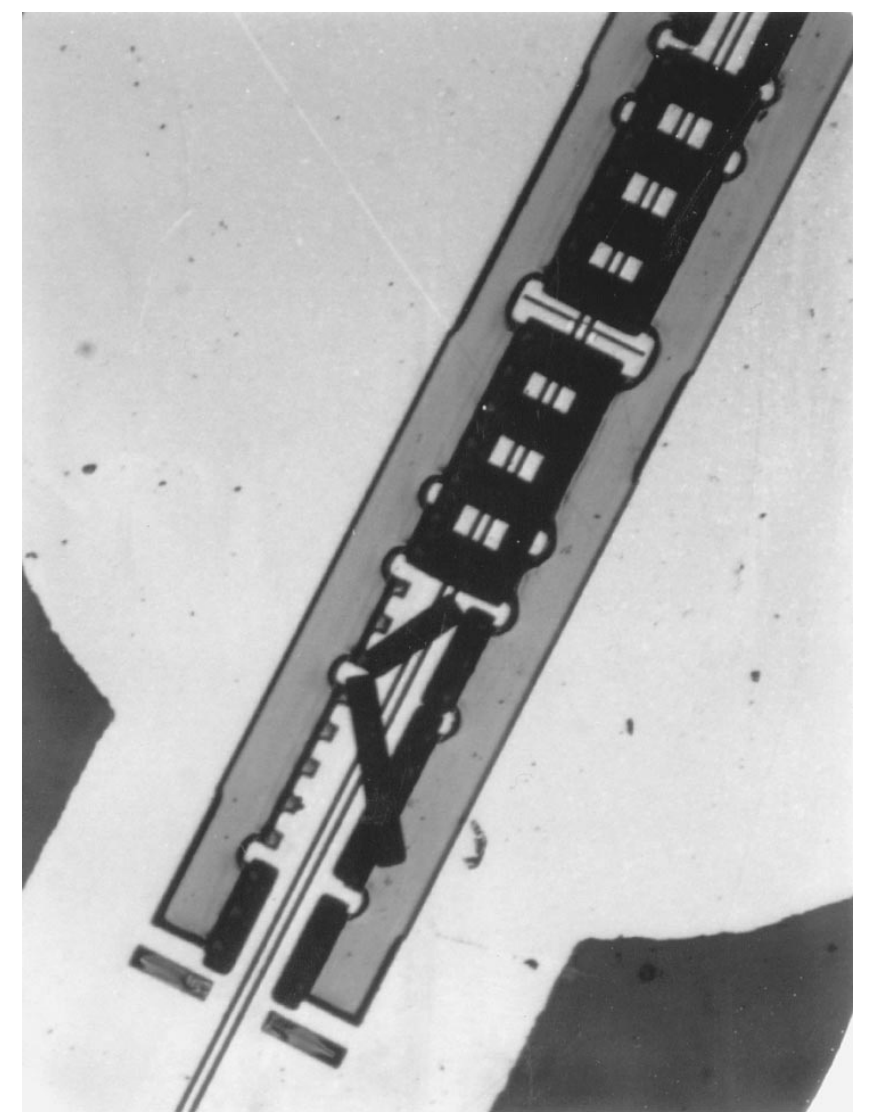

Fig. 4. Microscope photograph showing the removal of two of the sacrificial gold structures (angled bars) used to form the polyimide guides. Once these have all been removed, the SPB tuners can slide freely along the transmission line within the confines of the guides.

strips were then cured in an inert gas environment to form 9- $\mu$ m-thick polyimide guide structures.

Finally, the copper plating and sacrificial-seed layers were removed through wet etching to release the gold SPB and sacrificial structures. The sacrificial pieces shown in Fig. 4 were then removed from under the polyimide guides to allow the SPB structures to slide within the guides along the surface of the CPW lines. The digitated structure of the guides both minimized the chance of binding between the SPB and its guide and provided reference marks for positioning the tuners. Fig. 5(a) and (b) shows a scanning electron microscope (SEM) photograph of a guide structure with its confined SPB in view and one of a guide structure with its SPB removed, respectively. Fig. 6 shows a released SPB tuner in its entirety.

The fabrication of state-of-the-art submillimeter-wave integrated circuits typically requires constant painstaking attention, which is justified by the fact that a single functioning circuit can yield a great deal of valuable data. The circuit demonstrated here required a full wafer and seven processing layers and was no exception. Fabrication, however, was successfully carried out with very limited processing equipment and in facilities with little or no environmental regulation. While yield was not optimized or specifically evaluated, a developmental wafer processed with an array of ten test tuners yielded six elements, which could slide smoothly without sticking, and four damaged elements. Similarly, of the final six circuit wafers simultaneously processed to produce one suitable for 


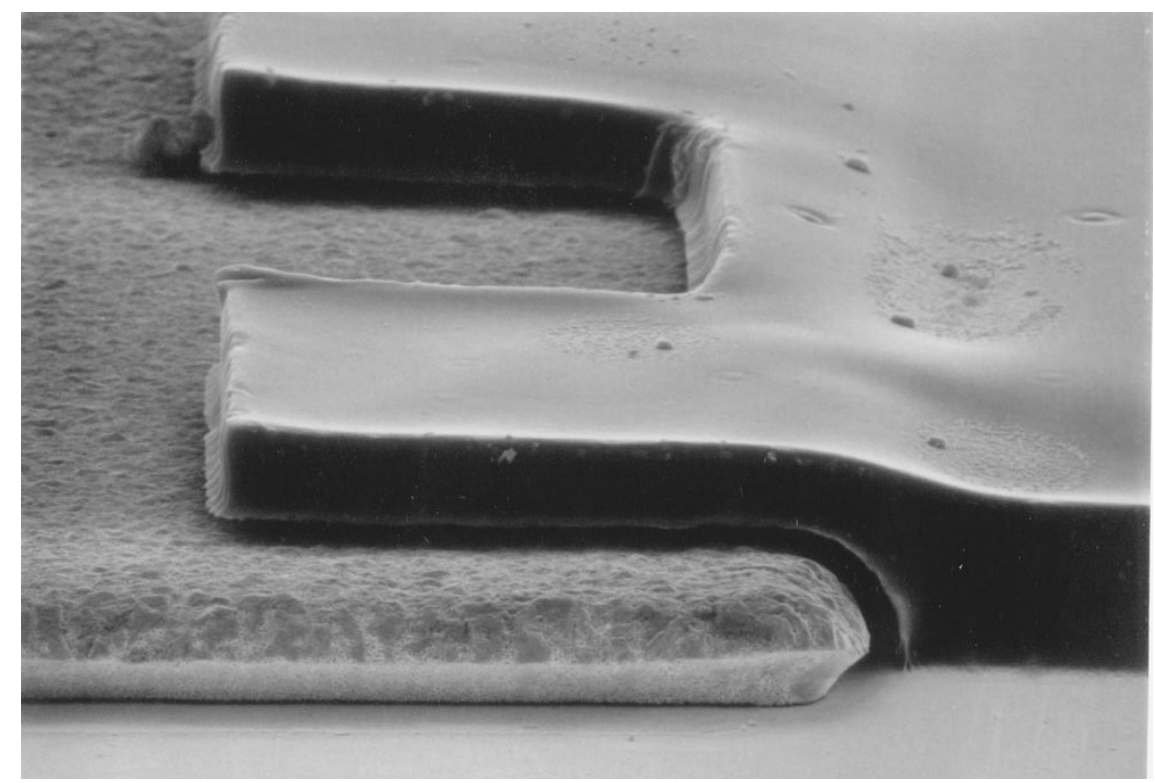

(a)

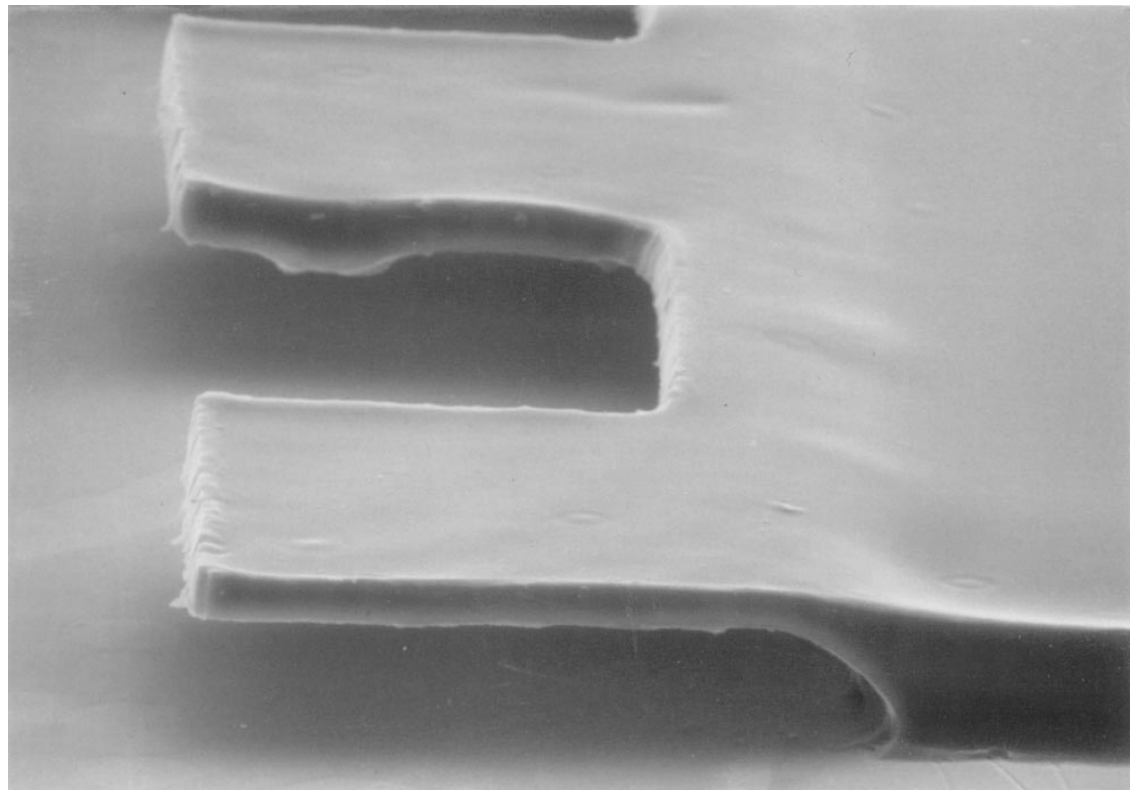

(b)

Fig. 5. Close-up SEM photograph of one side of an SPB tuner. (a) A polyimide guide structure is shown with a 5- $\mu$ m-thick SPB beneath it and (b) another with the SPB removed.

submillimeter-wave measurements, two exhibited tuner binding-one due to nonuniform gold plating and the other due to incomplete coverage of the electroplated sacrificial copper. The yield for producing a functioning submillimeter-wave bismuth detector in such a circuit is typically lower than that of these tuners.

\section{TUNER FUNCTION}

The processed wafer was mounted over a substrate lens to allow quasi-optical coupling to a $620-\mathrm{GHz}$ backward wave oscillator source. The performance of the integrated SPB tuners was demonstrated by using them to vary the power delivered from the slot antenna to the bismuth detector by altering the impedance match between them. The delivered power was measured using a lock-in amplifier, and a theoretical model was also derived to calculate the circuit behavior in order to predict and verify the measured performance [20].

The tuners were positioned manually using a probe with an ox-hair tip which provided adequate manipulation control for the $620-\mathrm{GHz}$ experiment. Ox hair was determined to be well suited for the application as it had sufficient stiffness for pushing the tuner, yet could safely brush against the polyimide and silicon dioxide surfaces without causing damage. Power measurements were made for various alignments of the tuners at $20-\mu \mathrm{m}$ increments, which is one sixteenth of a guided wavelength. The circuit was designed to accommodate movement of the tuners over three guided wavelengths, though in a practical circuit there would be no need for positioning 


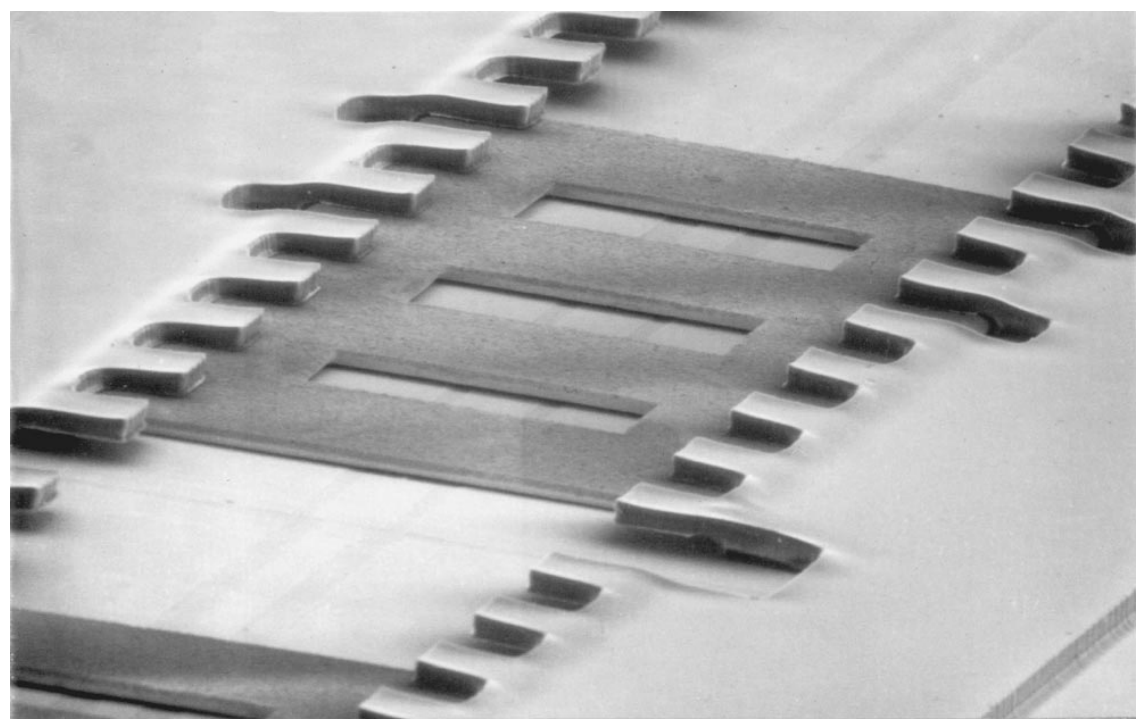

Fig. 6. SEM photograph of an integrated SPB tuner. An ox-hair probe was used to push each $200-\mu$ m-wide SPB along its guide to vary the electrical length of the CPW line beneath it.

the tuner beyond the first one-half guided wavelength as the performance pattern repeats after this, only with greater loss due to the added length of the transmission line. While over most of the range the tuners exhibited no sticking, positioning by successive $20-\mu \mathrm{m}$ increments was difficult and so the measurements were made for a more or less random order of tuner positions. The polyimide guides and stopping structures kept the tuners within the desired range at all times, and optimum circuit performance could be repeated without much difficulty. The submillimeter-wave circuit response was successfully varied over a $15-\mathrm{dB}$ range, which demonstrated adequate tuning capability for providing a good impedance match between a wide range of typical submillimeter-wave devices with high-parasitic reactances, such as SIS devices and Schottky diodes, and resonant planar antennas, including slots, dipoles, and self-complimentary structures. Measured results for independent position sweeps for each SPB tuner agreed very closely with theory and could be accurately repeated in tests conducted over a two-week period. The submillimeterwave function and performance of this circuit is described in more detail elsewhere [21], [22].

The fabrication techniques used for the tuner and all-planar circuitry are well suited to scaling the design to frequencies up to several terahertz. More precise positioning control may be necessary in such circuits and could be achieved through the use of mechanical manipulators such as those used for positioning optical fibers or through electrostatic [23], shapememory alloy [24], or other integrated actuator techniques. The sliding structure also has the potential to be used for other micromechanical millimeter- and submillimeter-wave circuit components such as switches, adjustable antenna elements, and aperture shutters.

\section{CONCLUSION}

A new submillimeter-wave tuning element has been developed along with a technique for its fabrication as an integral part of a monolithic circuit. The technique is based on silicon surface micromachining and LIGA, but incorporates only processes and materials suitable for common submillimeterwave integrated circuits. The performance of these tuning elements has been demonstrated at $620 \mathrm{GHz}$ through mechanical manipulation. This is the first reported demonstration of micromechanically adjustable tuning in a submillimeterwave integrated circuit. Potentially, the sliding element can be adapted to serve additional millimeter- and submillimeterwave circuit functions at frequencies up to several terahertz and, if necessary, could be made to self-actuate through the application of electrostatic, shape-memory alloy, or other microelectromechanical actuation techniques, with appropriate consideration made for individual circuit compatibility with thermal, electrostatic, and other environmental factors.

\section{ACKNOWLEDGMENT}

The authors would like to thank P. A. Stimson for his generous assistance with the submillimeter-wave aspects of this experiment, E. Kolawa and J. S. Reid for assistance with dielectric sputtering, and O. Borić-Lubecke for assistance with the manuscript.

\section{REFERENCES}

[1] W. Y. Ali-Ahmad, G. M. Rebeiz, H. Davé, and G. Chin, "802 GHz integrated horn antennas imaging array," Int. J. Infrared and Millimeter Waves, vol. 12, no. 5, pp. 481-486, May 1991.

[2] W. R. McGrath, C. K. Walker, M. Yap, and Y.-C. Tai, "Silicon micromachined waveguides for millimeter-wave and subillimeter-wave frequencies," IEEE Microwave Guided Wave Lett., vol. 3, pp. 61-63, Mar. 1993.

[3] T. M. Weller, L. P. B. Katehi, and G. M. Rebeiz, "A $250 \mathrm{GHz}$ microshield bandpass filter," IEEE Microwave Guided Wave Lett., vol. 5, pp. 153-155, May 1995.

[4] L.-S. Fan, Y.-C. Tai, and R. S. Muller, "Integrated moveable micromechanical structures for sensors and actuators," IEEE Trans. Electron Devices, vol. 35, no. 6, pp. 724-730, 1988.

[5] A. B. Frazier and M. G. Allen, "Metallic microstructures fabricated using photosensitive polyimide electroplating molds," IEEE J. Microelectromech. Syst., vol. 2, no. 2, pp. 87-94, 1993.

[6] I. Mehdi and P. H. Siegel, "Effect of parasitic capacitance on the performance of planar subharmonically pumped Schottky diode mixers," 
in 5th Int. Symp. Space Terahertz Technology—Symp. Proc., May 1994, pp. 379-393.

[7] R. Blundell and C.-Y. E. Tong, "Submillimeter receivers for radio astronomy," Proc. IEEE, vol. 80, no. 11, pp. 1702-1720, 1992.

[8] M. Salez, P. Febvre, W. R. McGrath, B. Bumble, and H. G. Leduc, "An SIS waveguide heterodyne receiver for $600 \mathrm{GHz}-635 \mathrm{GHz}$," Int. $J$. Infrared and Millimeter Waves, vol. 15, no. 2, pp. 349-368, Feb. 1994.

[9] J. Zmuidzinas, H. G. Leduc, J. A. Stern, and S. R. Cypher, "Two junction tuning circuits for submillimeter SIS mixers," IEEE Trans. Microwave Theory Tech., vol. 42, no. 4, pp. 698-706, 1994.

[10] R. E. Collin, Foundations for Microwave Engineering. New York: McGraw-Hill, 1966.

[11] V. M. Lubecke, W. R. McGrath, and D. B. Rutledge, "Sliding backshorts for planar circuits," Int. J. Infrared and Millimeter Waves, vol. 12, no. 12, pp. 1387-1397, Dec. 1991.

[12] _ "A $100 \mathrm{GHz}$ coplanar strip circuit tuned with a sliding planar backshort," IEEE Microwave Guided Wave Lett., vol. 3, pp. 441-443, Dec. 1993.

[13] K. C. Gupta, R. Garg, and I. J. Bahl, Microstrip Lines and Slotlines. Dedham, MA: Artech, 1979.

[14] V. M. Lubecke, W. R. McGrath, and D. B. Rutledge, "Millimeter wave performance of a sliding planar backshort," in SPIE Int. Conf. Millimeter and Submillimeter Waves and Applications, Jan. 1994, pp. 543-544.

[15] P. H. Siegel and R. J. Dengler, "The dielectric-filled parabola: A new millimeter and submillimeter wavelength receiver/transmitter front end," IEEE Trans. Antennas Propagat., vol. 39, no. 1, pp. 40-47, 1991.

[16] D. P. Neikirk and D. B. Rutledge, "Self-heated thermocouples for farinfrared detection," Appl. Phys. Lett., vol. 41, no. 5, pp. 400-402, Sept. 1992.

[17] I. Mehdi, T. H. Lee, D. A. Humphery, S. C. Martin, R. J. Dengler, J. E. Oswald, A. Pease, R. P. Smith, and P. H. Siegel, " 600 GHZ planarSchottky-diode subharmonic waveguide mixers," in 1996 IEEE MTT-S Int. Symp., June 1996, pp. 377-380.

[18] P. A. Stimson et al., "A planar quasioptical SIS receiver," IEEE Trans. Microwave Theory Tech., vol. 14, no. 4, pp. 609-615, 1993.

[19] V. M. Lubecke, W. R. McGrath, and D. B. Rutledge, "Micromechanical sliding planar backshorts," in Int. Semiconductor Device Research Symp., Charlottesville, VA, Dec. 7, 1995, pp. 477-480.

[20] V. M. Lubecke, "Micromechanical tuning elements for submillimeter wave integrated circuits," Ph.D. dissertation, Calif. Instit. Technol., Pasadena, CA, July 1995.

[21] V. M. Lubecke, W. R. McGrath, P. A. Stimson, and D. B. Rutledge, "Micromechanical tuning elements in a $620 \mathrm{GHz}$ integrated circuit," IEEE Trans. Microwave Theory Tech., to be published.

[22] _ "Performance of micromechanical tuning elements in a 620 GHz monolithic integrated circuit," in 7th Int. Symp. Space Terahert Technology, Charlottesville, VA, Mar. 1996, pp. 220-233.

[23] T. Akiyama and K. Shono, "Controlled stepwise motion in polysilicon microstructures," IEEE J. Microelectromech. Syst., vol. 2, no. 3, pp. 106-110, 1993.

[24] P. Krulevitch, A. P. Lee, P. B. Ramsey, J. C. Trevino, J. Hamilton, and M. A. Northrup, "Thin film shape memory alloy microactuators," IEEE J. Microelectromech. Syst., vol. 5, no. 4, pp. 270-282, 1996.

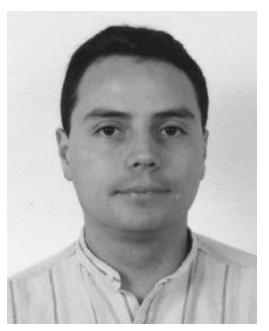

Victor M. Lubecke (SM'98) received the B.S. degree in electrical and electronic engineering from the California State Polytechnical University, Pomona, in 1986 and the M.S. and Ph.D. degrees in electrical engineering from the California Institute of Technology, Pasadena, in 1990 and 1995, respectively

His graduate work focused on high-frequency integrated-circuit techniques and microelectromechanical systems (MEMS) technology. From 1987 to 1996, he was with the NASA Jet Propulsion Laboratory working on millimeter- and submillimeter-wave technology in space communications and remote-sensing applications. Since 1996, he has been a Visiting Researcher at the Photodynamics Research Center, Institute for Physical and Chemical Research (RIKEN), Sendai, Japan. His current research interests include high-frequency integrated circuits, quasi-optics, and related MEMS techniques.
William R. McGrath (M'88) received the B.S. degree in physics from the Massachusetts Institute of Technology, Cambridge, in 1978 and the M.A. and $\mathrm{Ph} . \mathrm{D}$. degrees in physics from the University of California, Berkeley, in 1981 and 1985 , respectively.

His graduate work focused on the device physics of SIS mixers and superconductive circuits. He and his coworkers were the first to measure the quantum effects of large-gain and quantum-limited noise in SIS mixers at 36 GHz. Upon graduating from the University of California, Berkeley, he took a position as a Visiting Researcher at the Chalmers University of Technology, Goteborg, Sweden, where he worked on submillimeter-wave superconductive detectors. He and coworkers built the first millimeter-wave Josephson effect mixer using the newly discovered high-Tc superconductors. He joined the staff of the Jet Propulsion Laboratory, California Institute of Technology, Pasadena, CA, in 1987, where he became a Group Leader in 1990 and a Technical Group Supervisor in 1992. He heads a research group which develops highperformance submillimeter-wave sensors for remote-sensing applications. $\mathrm{He}$ has over 120 publications and two patents.

Dr. McGrath has received several awards related to his research activities.

Yu-Chong Tai received the B.S. degree in electrical engineering from the National Taiwan University, Taipei, Taiwan, R.O.C., in 1981 and the M.S. and Ph.D. degrees from the University of California, Berkeley, in 1986 and 1989, respectively.

$\mathrm{He}$ is currently an Associate Professor of Electrical Engineering, California Institute of Technology, Pasadena, where he directs the Caltech Micromachining Laboratory which currently sponsors more than 20 researchers for micromachining. He has over 12 years of experience doing micromachines and/or MEMS research. His research interests include MEMS technology, microsensors, microactuators, microstructures, MEMS systems, and MEMS science. He has successfully developed MEMS devices in his lab including pressure sensors, shear-stress sensors, hot-wire anemometers, magnetic actuators, microphones, microvalves, micromotors, etc. System-level MEMS research projects then include integrated M3 (microelectronics + microsensors + microactuators) drag-reduction smart surface, flexible smart skin for the control unmanned aerial vehicles, and microfluid delivery systems. He is also interested in MEMS sciences such as MEMS material (mechanical and thermal) properties, microfluid mechanics, and micro/nano processing issues.

David B. Rutledge (M'75-SM'89-F'93) received the B.A. degree in mathematics from the Williams College, Williamstown, MA, in 1973, the M.A. degree in electrical sciences from Cambridge University, Cambridge, U.K., in 1975, and the Ph.D. degree from the University of California, Berkeley, in 1980 .

He has been teaching at the California Institute of Technology, Pasadena, and working on microwave circuits and antennas since 1980. His research group developed key ideas in integrated-circuit antennas, including lenscoupled antennas, which appear widely in radio-astronomy receivers. His group demonstrated anisotropic etching for fabricating horns and membrane technology for suspending metal antennas. The group first described leakage from planar transmission lines and first demonstrated many active quasi-optical components, including phase shifters, oscillators, mixers, and amplifiers. Recently, the group has developed Class-E HF power amplifiers for industrial and amateur use. He has authored or coauthored over 200 publications. He is a coauthor along with S. Wedge and R. Compton of the widely distributed educational microwave computer-aided design package Puff, with 15000 copies worldwide. He was a Visiting Scientist at CSIRO, New South Wales, Australia, in the summer of 1985, the Research Institute for Electrical Communication, Tohoku University, Sendai, Japan, in the Spring and Summer of 1988, and the National Defense Academy, Yokosuka, Japan, in the Fall of 1995. He has been a Distinguished Lecturer for the Antennas and Propagation Society from 1991 to 1993 . He has served as Chairman for 19 doctoral candidates.

Dr. Rutledge is a Member of the AP-S AdCom. He received the Teaching Award of the Associated Students of Caltech. In 1993, he received the MTT-S Microwave Prize. 